Bull. Austral. Math. Soc.

VoL. $42(1990)$ [95-100]

\title{
THE NORMAL STRUCTURE OF JAMES \\ QUASI REFLEXIVE SPACE
}

\author{
Daryl Tingley
}

It is shown that weakly compact sets of James quasi reflexive space have normal structure.

\section{Normal Structure and FiXed points}

Let $K$ be a nonempty bounded convex subset of a Banach space $X$. The diameter of $K$, denoted $\operatorname{diam}(K)$, is defined by $\operatorname{diam}(K)=\sup \{\|x-y\|: x, y \in K\}$. (Here $K$ need not be convex.) The set $K$ is said to have normal structure if for every bounded convex set $H \subset K$ with $\operatorname{diam}(H)>0$, there is $x_{0} \in H$ such that $\operatorname{diam}(H)>$ $\sup \left\{\left\|x-x_{0}\right\|: x \in H\right\}$. Normal structure was introduced by Brodskii and Milman [2] and has since been used extensively (see [11] for a survey) in the study of fixed point properties of nonexpansive maps. (A map $T: K \rightarrow K$ is said to be nonexpansive if $\left\|T_{x}-T_{y}\right\| \leqslant\|x-y\|$ for all $x, y \in K$.) Indeed, Kirk [5] has shown that if $K$ is a weakly compact convex set that has normal structure, then every non-expansive map $T: K \rightarrow K$ has a fixed point. (However, Karlovitz [4] has shown that normal structure is not a necessary condition.)

The set $K$ is said to have the fixed point property (f.p.p.) if every nonexpansive map $T: K \rightarrow K$ has a fixed point. If every weakly compact set $K \subset X$ has the f.p.p., then $X$ is said to have the f.p.p. Thus $X$ has the f.p.p. if weakly compact sets have normal structure, in which case we say that $X$ has weak normal structure.

\section{JAMES QUASI-REFLEXIVE SPACE}

James space, $J$, consists of all the real sequences $\left(\alpha_{n}\right)$ for which $\lim \alpha_{n}=0$ and $\left\|\left(\alpha_{n}\right)\right\|_{J}<\infty$ where

$$
\left\|\left(\alpha_{n}\right)\right\|_{J}=\sup \left\{\left[\left(\alpha_{p_{1}}-\alpha_{p_{2}}\right)^{2}+\ldots+\left(\alpha_{p_{n-1}}-\alpha_{p_{n}}\right)^{2}+\left(\alpha_{p_{n}}-\alpha_{p_{1}}\right)^{2}\right]^{1 / 2}\right\}
$$

the supremum being taken over all finite increasing sequences of positive numbers $\left\{p_{1}, p_{2}, \ldots, p_{n}\right\}$. It is easily shown (see [9], Vol. I, p.25) that $J$ is a Banach space.

Received 12 September 1989

Copyright Clearance Centre, Inc. Serial-fee code: 0004-9729/90 \$A2.00+0.00. 
The space $J$ has been used to disprove several conjectures about Banach spaces [9] but perhaps its most striking property is that for which James [3] originally introduced it: $J$ is not reflexive, the canonical image of $J$ having codimension 1 in $J^{* *}$, but $J$ is nevertheless isometrically isomorphic to $J^{* *}$.

If the term $\left(\alpha_{p_{n}}-\alpha_{p_{1}}\right)^{2}$ is dropped from (1), another Banach space, sometimes called $J_{1}$, is obtained. $J_{1}$ and $J$ are isomorphic, however, the norm $\|\cdot\|_{J}$ is needed to show that $J$ is isometric and not just isomorphic to $J^{* *}$. In [6] Khamsi showed that weakly compact sets in $J_{1}$ have normal structure and hence $J_{1}$ satisfies the f.p.p. He mentions there that it is unclear whether or not $J$ has weak normal structure. In [7], Khamsi shows that $J$ also has the f.p.p. The method used does not depend on normal structure and indeed was developed to show certain spaces that lack normal structure have the f.p.p. $[1,8,10]$.

In this paper we show in a direct fashion that $J$ has weak normal structure. Combining this with the theorem of Kirk, this gives another proof that $J$ has the f.p.p.

3.

We say that a Banach space satisfies property $\left({ }^{*}\right)$ if every sequence $\left(x_{n}\right)$ that converges weakly to 0 satisfies

(*) $^{*} \sup _{m}\left\{\limsup _{n}\left\|x_{m}-x_{n}\right\|\right\}>\liminf _{n}\left\|x_{n}\right\|$.

It is easily seen that $\left({ }^{*}\right)$ is equivalent to

(*') $\sup \left\{\limsup \left\|x-x_{n}\right\|: x \in \operatorname{co}\left(x_{1}, x_{2}, \ldots\right)\right\}>\liminf _{n}\left\|x_{n}\right\|$, where $\operatorname{co}\left(x_{1}, x_{2}, \ldots\right)$ is the convex hull of the set $\left\{x_{n}\right\}$.

TheOREM 1. If a Banach space $X$ satisfies property $\left({ }^{*}\right)$, then $X$ has weak normal structure.

Proof: Suppose that $K \subset X$ is weakly compact and does not have normal structure. Then a result of Brodskii and Milman [2] shows that there is a sequence $\left\{x_{n}\right\} \subset K$ such that

$$
\lim d\left(x_{n+1}, \operatorname{co}\left(x_{1}, \ldots, x_{n}\right)\right)=\operatorname{diam}\left\{x_{n}\right\},
$$

where $\operatorname{diam}\left\{x_{n}\right\}=\sup \left\{\left\|x_{n}-x_{m}\right\|: n, m \geqslant 1\right\}$ and

$$
d\left(x_{n+1}, \operatorname{co}\left(x_{1}, \ldots, x_{n}\right)\right)=\inf \left\{\left\|x_{n+1}-x\right\|: x \in \operatorname{co}\left(x_{1}, \ldots, x_{n}\right)\right\} .
$$

Since any subsequence of $\left\{x_{n}\right\}$ also satisfies (2), and since both (2) and normal structure are invariant under translations, we may suppose that $\left\{x_{n}\right\}$ converges weakly to $0 \in K$. From (2) it follows that $\lim \left\|x-x_{n}\right\|=\operatorname{diam}\left\{x_{n}\right\}$ for all $x \in \operatorname{co}\left(x_{1}, x_{2}, \ldots\right)$ and hence it is easily seen that $\left\{x_{n}\right\}$ does not satisfy $\left({ }^{*}\right)$. 
Although I have no counterexample, it would seem unlikely that $\left(^{*}\right)$ is equivalent to weak normal structure for a Banach space $X$.

The following provides a partial converse of Theorem 1 and indicates why counterexamples are not so easy to find.

Theorem 2. Suppose that a Banach space $X$ has weak normal structure and satisfies the so called Opial condition (see [11], p.213): For every sequence $\left\{x_{n}\right\}$ that converges weakly to 0 , and for every $x \in X$,

$$
\liminf \left\|x-x_{n}\right\| \geqslant \liminf \left\|x_{n}\right\| \text {. }
$$

Then $X$ satisfies $\left(^{*}\right)$.

REMARK. Any Banach space that has an unconditional basis with unconditional basis constant 1 (see [9]) satisfies the Opial condition.

Proof: Suppose not. Then (from $\left({ }^{* \prime}\right)$ ) there is a sequence $\left\{x_{n}\right\}$ that converges weakly to 0 such that

$$
\sup \left\{\limsup \left\|x-x_{n}\right\|: x \in \operatorname{co}\left(x_{1}, x_{2}, \ldots\right)\right\} \leqslant \liminf _{n}\left\|x_{n}\right\|
$$

Combining (3) and (4) it follows that

$$
\lim _{n}\left\|x-x_{n}\right\|=\liminf _{n}\left\|x_{n}\right\| \text { for every } x \in \operatorname{co}\left(x_{1}, x_{2}, \ldots\right)
$$

Thus $X$ does not have weak asymptotic normal structure (see [11], pp.209, 210). Since weak asymptotic normal structure and weak normal structure are equivalent [11], this contradicts the hypothesis that $X$ has weak normal structure.

Theorem 3. The Banach space $J$ satisfies condition $\left({ }^{*}\right)$ and hence has weak normal structure and the fixed point property.

Proof: Let $\left\{x_{n}\right\} \subset J$ be any sequence that converges weakly to 0 . Notice that if any subsequence of $\left\{x_{n}\right\}$ satisfies $\left(^{*}\right)$, then $\left\{x_{n}\right\}$ satisfies $\left(^{*}\right)$.

Let $e_{n}=(0, \ldots, 0,1,0, \ldots)$ where the 1 is in the $n$th position. Then $\left\{e_{n}\right\}$ is a basis for $J([9], p .25)$ and we may write $x_{n}=\sum_{i=1}^{\infty} \alpha_{i}^{n} e_{i}$ for each $n$. Since $\left\{x_{n}\right\}$ converges weakly to $0, \alpha_{i}^{n} \underset{n}{\rightarrow} 0$ for each $i$. Thus, possibly by extracting a subsequence of $\left\{x_{n}\right\}$, we may choose a sequence of integers $\left\{b_{n}\right\}$ such that if $u_{n}=\sum_{i=n+1}^{b_{n}} \alpha_{i}^{n} e_{i}$ then

$$
\left\|x_{n}-u_{n}\right\| \rightarrow 0
$$


Write $u_{n}=\sum_{i=1}^{\infty} \beta_{i}^{n} e_{i}$. Obviously $\beta_{i}^{n}=\alpha_{i}^{n}$ for $n+1 \leqslant i \leqslant b_{n}$ and $\beta_{i}^{n}=0$ otherwise. (In particular $\beta_{n}^{n}=0$.) For each $n$, only finitely many $\beta_{i}^{n}$ are non-zero so the supremum in (1) is obtained for some finite sequence $p_{1}, \ldots, p_{k}$. That is

$$
\left\|u_{n}\right\|^{2}=\sum_{i=1}^{k}\left(\beta_{p_{i}}^{n}-\beta_{p_{i+1}}^{n}\right)^{2}+\left(\beta_{p_{k}}^{n}-\beta_{p_{1}}^{n}\right)^{2} .
$$

The set $\left\{p_{i}\right\}_{i=1}^{k}$ depends on $n$. It is understood that when working with $u_{n}$ the corresponding $\left\{p_{i}\right\}_{i=1}^{k}$ is being used. The set $\left\{p_{i}\right\}$ may be chosen so that none of the terms in (6) is 0 and

$$
n \leqslant p_{1} \leqslant \ldots \leqslant p_{k} \leqslant b_{n}
$$

If both $\beta_{p_{1}}^{n}$ and $\beta_{p_{k}}^{n}$ are non-zero they must have different signs, for otherwise $p_{1} \neq n$ and the indices $n, p_{1}, \ldots, p_{k}$ show that $\left\|u_{n}\right\|$ is greater than that given by (6). By one final extraction of a subsequence of $\left\{x_{n}\right\}$ (and the corresponding subsequences of $\left\{u_{n}\right\}$ and $\left\{b_{n}\right\}$ ) we suppose that either

$$
\beta_{p_{1}}^{n} \leqslant 0 \leqslant \beta_{p_{k}}^{n}
$$

or $\beta_{p_{k}}^{n} \leqslant 0 \leqslant \beta_{p_{1}}^{n}$ for all $n$. As the proofs are similar, it is assumed that (8) holds.

We have now constructed sequences $\left\{x_{n}\right\}$ and $\left\{u_{n}\right\}$ so that $(5)-(8)$ hold.

Let

$$
M=\sup \left\{\alpha_{i}^{n}: i \geqslant 1, n \geqslant 1\right\}, \quad m=\inf \left\{\alpha_{i}^{n}: i \geqslant 1, n \geqslant 1\right\} .
$$

Notice that $m \leqslant \beta_{i}^{n} \leqslant M$ for each $i$ and $n, m \leqslant 0 \leqslant M$, and at least one of $m$ and $M$ is non-zero. We have two cases to consider.

$$
M \geqslant-m \text { and } M>0
$$

or $-m \geqslant M$ and $-m>0$.

CASE I. $M \geqslant-m$ and $M>0$.

Let $\varepsilon, 0<\varepsilon<M$ be given, and let $q, t$ satisfy

$$
\alpha_{t}^{q}>M-\varepsilon
$$

Let $r>t$ be arbitrary and let $s=r-1$. Then

$$
\left\|x_{q}-x_{r}\right\| \geqslant\left\|P_{s} x_{q}-u_{r}\right\|-\left\|\left(I-P_{s}\right) x_{q}\right\|-\left\|u_{r}-x_{r}\right\| .
$$


(Here $\left.P_{s} x_{q}=\alpha_{1}^{q} e_{1}+\ldots+\alpha_{q}^{q} e_{d}\right)$.

The indices $t, r, p_{1}, \ldots, p_{k}$ show that (recall $\beta_{r}^{r}=0$ )

$$
\begin{aligned}
\left\|P_{s} x_{q}-u_{r}\right\|^{2} & \geqslant\left(\alpha_{t}^{q}\right)^{2}+\left(\beta_{p_{1}}^{r}\right)^{2}+\left\|u_{r}\right\|^{2}-\left(\beta_{p_{k}}^{r}-\beta_{p_{1}}^{r}\right)^{2}+\left(\beta_{p_{k}}^{r}+\alpha_{t}^{q}\right)^{2} \\
& =\left\|u_{r}\right\|^{2}+2 \beta_{p_{k}}^{r}\left(\beta_{p_{1}}^{r}+\alpha_{t}^{q}\right)+2\left(\alpha_{t}^{q}\right)^{2} .
\end{aligned}
$$

From (8), (9), (10) and (11)

$$
\left\|P_{,} x_{q}-u_{r}\right\|^{2} \geqslant\left\|u_{r}\right\|^{2}-2 M \varepsilon+2(M-\varepsilon)^{2} .
$$

Thus (12) becomes

$$
\left\|x_{q}-x_{r}\right\| \geqslant \sqrt{\left\|u_{r}\right\|^{2}-2 M \varepsilon+2(M-\varepsilon)^{2}}-\left\|\left(I-P_{\odot}\right) x_{q}\right\|-\left\|u_{r}-x_{r}\right\| .
$$

Since $r$ can be arbitrarily large and $s=r-1$ taking limits in $r$ gives

$$
\underset{r}{\limsup }\left\|x_{q}-x_{r}\right\| \geqslant \sqrt{\limsup \left\|u_{r}\right\|^{2}-2 M \varepsilon+2(M-\varepsilon)^{2}} .
$$

Since $\varepsilon>0$ was arbitrary,

$$
\sup _{q}\left\{\limsup _{r}\left\|x_{q}-x_{r}\right\|\right\} \geqslant \sqrt{\limsup _{r}\left\|u_{r}\right\|^{2}+2 M^{2}}=\sqrt{\limsup _{r}\left\|x_{r}\right\|^{2}+2 M^{2}}
$$

and since $M>0,\left\{x_{n}\right\}$ satisfies $\left({ }^{*}\right)$.

CASE II. $M<-m$ and $m<0$.

Let $\varepsilon$ be given with $0<\varepsilon<-m$, and choose $q, t$ so that $\alpha_{t}^{q}<m+\varepsilon$. Let $r$ be such that $t<r$ and let $s=r-1$. Then (12) is satisfied and in a manner similar to Case $\mathrm{I}$, the indices $t, p_{1}, \ldots, p_{k}, b_{r}+1$ show that

$$
\left\|P_{s} x_{q}-u_{r}\right\|^{2} \geqslant\left\|u_{r}\right\|^{2}+2(m+\varepsilon)^{2}+2 m \varepsilon .
$$

Substituting this into (12) it follows that

$$
\sup _{q}\left\{\limsup \left\|x_{q}-x_{r}\right\|\right\} \geqslant \sqrt{\limsup _{r}\left\|x_{r}\right\|^{2}+2 m^{2}}
$$

and hence $\left\{x_{n}\right\}$ satisfies $(*)$.

Since $\left\{x_{n}\right\} \subset J$ was an arbitrary sequence converging weakly to 0 , we have shown that $J$ satisfies property $\left({ }^{*}\right)$. 


\section{REFERENCES}

[1] J.M. Borwein and B. Sims, Nonexpansive mappings on Banach lattices and related topics', Houston J. Math. 10 (1984), 339-355.

[2] M.S. Brodskii and D.P. Milman, 'On the center of a convex set', Dokl. Akad. Nauk SSSR 59 (1948), 837-840.

[3] R.C. James, 'A non-reflexive Banach space isometric with its second conjugate space', Proc. Nat. Acad. Sci. U.S.A. 37 (1951), 134-177.

[4] L.A. Karlovitz, 'Existence of fixed points for nonexpansive mappings in a space without normal structure', Pacific J. Math. 66 (1976), 153-159.

[5] W.A. Kirk, 'A fixed point theorem for mappings, which do not increase distances', Amer. Math. Monthly 72 (1965), 1004-1006.

[6] M.A. Khamsi, 'Normal structure for Banach spaces with Schauder decomposition', Canadian Math. Bull. 32 (1989), ??-??.

[7] M.A. Khamsi, 'James quasi reflexive space has the fixed point property', Bull. Austral. Math. Soc. 39 (1989), 25-30.

[8] P.K. Lin, 'Unconditional bases and fixed points of nonexpansive mappings', Pacific J. Math. 116 (1985), 69-76.

[9] J. Lindenstrauss and L. Tzafriri, Classical Banach spaces, Vol. I and II (Springer-Verlag, Berlin, Heidelberg, New York, 1977 and 1979).

[10] B. Maurey, Points fixes des contractions sur un convexe ferme de $L_{1}$ : Seminaire d'analyse fonctionelle (Ecole Polytechnique, Palaiseau, Exposé No. VIII, 1980/81).

[11] S. Swaminathan, 'Normal structure in Banach spaces and its generalization', Contemp. Math. 18, 201-215. (A.M.S., Providence, R.I.).

Department of Mathematics and Statistics

University of New Brunswick

Fredericton, N. B.

Canada E3B 5A3 